\title{
Metformin does not Reduce Inflammation in Diabetics with AAA or at High Risk of AAA Formation
}

\author{
S. Keisin Wang, Linden A. Green, Ashley R. Gutwein, Bianca Kenyon, Raghu L. Motaganahalli, Andres \\ Fajardo, Alok K. Gupta, Michael P. Murphy
}

\section{Introduction:}

The protective effect of diabetes mellitus (DM) on abdominal aortic aneurysm (AAA) formation and growth has been repeatedly observed in population studies but continues to be poorly understood. However, recent investigations have suggested that metformin, a staple antihyperglycemic medication, may be independently protective against AAA formation and growth. Therefore, we describe the effect of metformin in AAA and at-risk patients on markers of inflammation, the driver of early AAA formation and growth.

\section{Methods:}

Peripheral blood was collected from patients previously diagnosed with AAA or presenting for their U.S. Preventive Task Force- recommended (USPTF) AAA screening. Plasma and circulating peripheral blood mononuclear cells (PBMCs) were isolated using ficoll density centrifugation. Circulating plasma inflammatory and regulatory cytokines were assessed with enzyme-linked immunosorbent assays (ELISA). $\mathrm{CD}^{+}$cell phenotyping was performed using flow cytometric analysis and expressed as a proportion of total $\mathrm{CD}^{+}$cells. To determine the circulating antibody to self-antigen response, a modified ELISA was performed against antibodies to collagen type V (COLV) and elastin fragments (ELNf).

\section{Results:}

Peripheral blood was isolated from 266 patients without DM (n=182), with DM not treated with metformin $(n=34)$, and with DM actively taking metformin $(n=50)$ from 2015-2017. We found no differences in the expression of $\operatorname{Tr} 1$, Th17, and Treg $\mathrm{CD} 4^{+}$fractions within diabetics \pm metformin. When 
comparing inflammatory cytokines, we detected no differences in IL-1 $\beta$, IL-6, IL-17, IL-23, IFN- $\gamma$, and TNF- $\alpha$. Conversely, no differences were observed pertaining to the expression to regulatory cytokines IL-4, IL-10, IL-13, TSG-6, or TGF- $\beta$. Lastly, no differences in expression of COLV and ELNf antigen and/or antibodies were detected with metformin use in diabetics.

\section{Conclusion:}

Metformin in diabetics at-risk for AAA or diagnosed with AAA does not seem to alter the peripheral inflammatory environment.

Keywords: Abdominal Aortic Aneurysm, Inflammation, Diabetes, Metformin 


\section{Background}

AAA is an major source of morbidity and mortality in the Western Hemisphere affecting as many as $4 \%$ of men starting their sixth decade of life.(1) This pathology is characterized by early local inflammation causing degradation of the arterial wall and progressive dilation until increasing wall tension eventually results in rupture.(2-4) Of those experiencing a catastrophic rupture event, approximately half die immediately and never present to the hospital; overall, short-term rupture mortality approaches $80 \% .(5,6)$ In the United States alone, over 15,000 deaths per annum are directly related to AAA rupture making it the number 11 killer of Americans.(7)

The gold standard of AAA care continues to be rupture prevention via aneurysm resection and aortic reconstruction when cross-sectional diameter exceeds $5.5 \mathrm{~cm} .(8)$ As a result, over 40,000 highlymorbid aortic reconstructions are performed annually resulting in health care costs in excess of $\$ 116,000$ per case in the perioperative period. $(9,10)$ Therefore, the magic bullet for AAA continues to be the elusive pharmaceutical which can slow or stop diameter growth.

Previous population studies have suggested a protective effect of DM on AAA formation and growth. $(6,11-13)$ Only recently has metformin been implicated as a potential cause of the AAAprivileged nature of the diabetic patient.(14,15) Our group has previously published studies suggesting the existence of an antigen-specific response unique to AAA subjects to breakdown products of elastin and collagen which may drive both immune aberrations leading to faulty regulation, via $\operatorname{Tr} 1$ and $\operatorname{Treg}$ dysfunction, and runaway inflammation, via Th17 overexpression.(16-19) Therefore, the purpose of this investigation was to establish the presence of cytokine changes, if any, in a population of AAA and highrisk patients associated with metformin use which may explain the potentially protective effect of metformin. 


\section{Methods}

Blood Banking and Isolation of PBMCs and Plasma

After the appropriate approval was obtained from the Indiana University (IU) Institutional Review Board (IRB \#1408881234), peripheral blood was collected from individuals giving informed consent at IU School of Medicine affiliated hospitals from 2015 to 2017. AAA-positive samples (>30 $\mathrm{mm}$ ) were collected from individuals presenting for follow-up of previously diagnosed aneurysms while samples from at-risk subjects (risk-factor matched, RFM) were isolated from patients who screened negative for their USPTF recommended AAA ultrasounds at age 65.(20) Mononuclear cells were isolated by standard ficoll density separation using Accuspin tubes (Sigma) as described in detail elsewhere.(17) Isolated PBMCs and plasma were stored at $-80^{\circ} \mathrm{C}$ in small aliquots to minimize freeze/thaw cycles.

CD4 $4^{+}$Lymphocyte Phenotyping

Cell staining using antibodies against identifying surface markers to $\operatorname{Tr} 1$, Th17, and Treg subsets of $\mathrm{CD}^{+}$lymphocytes were performed per manufacturer's instructions (1:10, Miltenyi) unless otherwise noted. Tr1: CD4-FITC, CD49b-PE (1:20), LAG3-APC (1:20); Treg: CD4-FITC, CD25-PE, FOXP3APC; Th17: CD4-FITC, CD194-PE, CD196-APC. Tr1 lymphocytes were stained for 15 minutes at room temperature while the remaining subsets were stained for 10 minutes at $4{ }^{\circ} \mathrm{C}$. Flow cytometric analysis was performed on an Accuri C6 (BD) with CellQuest software (BD).

Plasma Cytokine and Antigen Quantification 
Cytokine concentration in the plasma was determined using commercially available ELISA kits and performed per manufacturer's recommendations. Kits, when available, were obtained from R\&D; if a cytokine kit was not available via $R \& D$, an alternative was sourced from Sigma. To calculate absolute plasma concentrations, absorbance was read with a Cytation 5 (Biotek) at $450 \mathrm{~nm}$ and referenced to a standard curve.

\section{Plasma Antibody Quantification}

A modified ELISA was performed to determine relative concentrations of antibodies specific to COLV and ELNf. Human COLV (Sigma) and Elastin (Elastin Products Company) peptides were dissolved in phosphate buffered saline to a stock working solution of $25 \mathrm{ug} / \mathrm{mL}$. This solution was used to coat a high protein binding $96-$ well polystyrene plate (Sigma) overnight at $4{ }^{\circ} \mathrm{C}$. A blocking step was performed with a solution of $1 \%$ BSA for 2 hours at $37{ }^{\circ} \mathrm{C}$ or overnight at $4{ }^{\circ} \mathrm{C}$. Plasma samples were sequentially diluted up to 1:1000 to determine optimal concentration and incubated for 2 hours at room temperature. A goat anti-human IgG Fc antibody conjugated to horseradish peroxidase (HRP, Sigma) was utilized as a secondary antibody per manufacturer's recommended dilution. Reactions were performed using a 1-step TMB turbo substrate (Sigma) for 30 minutes before a $1 \mathrm{M}$ sulfuric acid stop solution was added. Absorbance was measured at $450 \mathrm{~nm}$ to determine relative intensity.(21) 


\section{Results}

\section{Baseline Comorbidities and Medications}

From June 2015 to September of 2017, a total of 266 patients contributed blood to the biorepository utilized for this study. All patients, regardless of AAA status, were divided into three cohorts, 1) no DM ( $\mathrm{n}=182), 2) \mathrm{DM}$ without metformin ( $\mathrm{n}=34)$, and 3) DM with metformin $(\mathrm{n}=50)$. The

baseline comorbidities of these cohorts are detailed in table 1. We observed no difference with respect to age between cohorts $(p=0.98)$. In terms of Framingham Risk Scores, nondiabetics had the lowest risk (33.1\%) compared to a higher risk associated with DM regardless of metformin use (49.4\% vs 49.8\%). Our three cohorts were homogeneous in terms of AAA, chronic obstructive pulmonary disease (COPD), hyperlipidemia (HLD), coronary artery disease (CAD), and AAA familial history (FHx) incidence. However, heterogeneity was encountered with respect to the incidence of hypertension (HTN), peripheral arterial disease $(\mathrm{PAD})$, chronic kidney disease $(\mathrm{CKD})$, and obesity $(\mathrm{BMI}>30)$. Baseline medication profiles are described in detail in table 2. Although a significant difference in the usage of angiotensinreceptor blockers (ARB) was noted, this effect disappeared when the prevalence of angiotensinconverting enzyme inhibitors (ACEi) were combined. Except for aspirin use, medication homogeneity was encountered across all cohorts.

CD4 $4^{+}$Lymphocyte Subsets

$\mathrm{CD}^{+}$lymphocytes of interest consisted of the regulatory $\operatorname{Tr} 1$ and Treg fractions and the inflammatory Th17 phenotype. We previously noted that patients with AAA demonstrated significantly decreased expression of the regulatory $\operatorname{Tr} 1(7.3 \%$ vs $1.4 \%, p<0.01)$ and $\operatorname{Treg}(2.6 \%$ vs $1.5 \%, p=0.05)$ lymphocytes compared to their RFM counterparts. $(4,17)$ Additionally, a strong trend suggesting an 
increase in the expression of the inflammatory Th17 cell was observed in the patients diagnosed with AAA. Therefore, we investigated whether the expression of these cell types normalized with the presence of metformin. In the diabetics, we did not observe a change in the $\operatorname{Tr} 1(3.0 \%$ vs $5.0 \%, p=0.24)$, Th17 (3.0\% vs $2.9 \%, p=0.92)$, or Treg $(1.6 \%$ vs $1.2 \%, p=0.47)$ fractions with the presence of metformin which would suggest a beneficial effect on decreasing inflammation (Figure 1).

\section{Inflammatory Cytokines}

The effect of metformin on the inflammatory cytokines OPN, IL-1 $\beta$, IL-6, IL-17, IFN- $\gamma$, TNF- $\alpha$, and IL-23 were examined. The presence of DM did not seem to significantly alter the inflammatory environment compared to nondiabetics. Although weak trends towards decreased concentrations of OPN (9.1 vs $6.3 \mathrm{ng} / \mathrm{mL}, p=0.34)$, IL-1 $\beta$ (4.4 vs $3.9 \mathrm{ng} / \mathrm{mL}, p=0.45)$, and IFN- $\gamma(18.4 \mathrm{vs} 9.2 \mathrm{ng} / \mathrm{mL}, p=0.24)$ were seen, no statistically significant reduction in inflammatory cytokines were confirmed with the introduction of metformin to diabetics (Figure 2).

\section{Regulatory Cytokines}

The regulatory cytokines TGF- $\beta$, IL-4, IL-10, IL-13, and TSG-6 were used as markers of inflammation suppression (Figure 3). While IL-4 concentration increased in response to metformin (2.7 vs $5.1 \mathrm{ng} / \mathrm{mL})$, this did not reach statistical significance $(p=0.23)$. In the remaining regulatory cytokines examined, no trends were noted to suggest an antiinflammatory effect of metformin in diabetics taking metformin.

COLV and ELNf Antigen and Antibody 
In our previous experiments, we noted significantly higher concentrations of both ELNf/COLV antigen and antibodies in circulation of RFM and AAA patients compared to healthy controls. However, we did not observe an inflammation suppressing effect of metformin on circulating COLV (0.73 vs 0.62 $\mathrm{ng} / \mathrm{mL}, p=0.56)$ or ELNf(15.1 vs $14.8 \mathrm{ng} / \mathrm{mLs}, p=0.94)$ antigens. Between all cohorts, plasma antigen levels were lowest in the nondiabetic patients. Similarly, antibodies specific to COLV (0.42 vs 0.38 Relative Units, $p=0.76)$ and ELNf ( 0.22 vs 0.20 RUs, $\mathrm{p}=0.78)$ did not differ with the introduction of metformin. With respect to both peptides, a slight nonsignificant increase was observed in circulating antigens and antibodies with the presence of DM compared to patients without DM. 


\section{Discussion}

We established the IU Center for Aortic Disease biorepository in 2015 as part of a concerted effort to stimulate research into the immune component of AAA formation and growth. Since the beginning of this initiative, we have collected blood from over 330 individuals. With the assistance of this growing collection, multiple anomalies in the human immune response that characterizes the AAA condition have been described. $(17,19)$

The negative association between DM and AAA has been observed and noted in numerous population-based studies over the previous two decades.(15) A recent meta-analysis pooled 13 population, prospective cohort, and case-control studies and reported an odds ratio of 0.59 (95\% CI, 0.52 $-0.67 ; p<0.01)$ in support of the protective effect of DM on AAA formation.(22) Another similar review identified 17 large population studies and observed an odds ratio of $0.80(0.70-0.90 ; p<0.01)$ between DM and AAA once again implicating DM as a protective factor.(12) It is not clear how DM attenuates AAA formation; however, animal models of hyperglycemia have demonstrated evidence of decreased infiltration of macrophages, elastolysis, and neovascularization of the aortic wall which are all hallmark of early AAA formation.(23) Additionally, the diabetic environment may also favorably alter vascular smooth muscle cell physiology, increase advanced glycation end-products, down-regulate matrix metalloproteinases, and decrease fibrinolysis causing an overall increase in aortic structural integrity.(24)

With the increasing drive to establish a pharmaceutical treatment for AAA, the role of medications associated with DM have been scrutinized in detail.(25) These initial studies supported a protective effect of metformin, thiazolidinediones, and sulfonylureas not observed in $\alpha$-glucosidase and DPP-4 inhibitors.(25) While the effect of thiazolidinediones and sulfonylureas have been called into question by some studies claiming little protective effect, metformin seemed to be particularly effective at 
both prevention and progression of AAA across multiple clinical investigations. $(14,26)$ It is thought that metformin functions to decrease circulating glucose by inhibiting hepatic gluconeogenesis and blunting the effect of glucagon via inhibition of mitochondrial complex I and inactivation of the protein kinase A (PKA) and cyclic adenosine monophosphate (cAMP) signaling pathway.(27) This effect also results in an increase in overall sensitivity to circulating insulin. While metformin's AAA-protective mechanism continues to be nebulous, suggestions of a pleiotropic effect consisting of the inhibition of extracellular matrix (ECM) remodeling, downregulation of inflammation, and reduction of oxidative stress have been made.(14, 28-31)

We did not observe a decrease in inflammation with metformin use in a combined cohort of atrisk and AAA patients. However, when the population is divided into AAA and non-AAA patients by metformin status, no differences are once again seen with respect to $\mathrm{CD} 4^{+}$lymphocyte, cytokine, antigen, and antibody expression.

We previously reported significant aberrations between the RFM and AAA populations with respect to $\mathrm{CD}^{+}$fractions of lymphocytes in circulation. $(17,19)$ In particular, there is a depletion of the $\operatorname{Tr} 1\left(\mathrm{CD}^{+} \mathrm{LAG}^{+} \mathrm{CD} 4 \mathrm{~b}^{+}\right)$regulatory lymphocyte in the AAA condition.(17-19) This inflammation suppressing cell is unique in the immune response as it is antigen specific, elaborating the regulatory cytokine IL-10 and eliminating myeloid derived antigen-presenting cells via the granzyme B pathway in response to antigen recognition and activation to suppress local inflammation.(32) Not surprisingly, we also noted a reduction of circulating IL-10 in AAA patients corresponding to the aforementioned loss of Tr1 activity.(17) In this study, we did not observe an antiinflammatory effect of metformin on IL-10 or Tr1 expression towards levels seen in the RFM population. Additionally, no therapeutic effect was seen in the inflammatory Th17 or regulatory Treg population towards "healthy" levels. 
Previous reports suggest that circulating elastin and collagen type V peptides may be significant antigens in the pathogenesis of COPD, an inflammatory and destructive disease of the pulmonary system and strong risk factor for AAA formation. $(21,33,34)$ In our AAA population, we noted increased expression of both soluble elastin antigen and antibodies circulating in the peripheral plasma of AAA patients compared to RFM controls.(4) We believe these two antigens may play a major role in driving the self-sensitization of inflammatory immune cells to the native infrarenal aortic wall as they predictably increase from healthy, to RFM, and finally AAA-diagnosed subjects.(4) Once again, our investigation did not demonstrate a decrease with metformin in the circulation of these inflammatory peptides in the diabetic population.

Although previous metformin studies suggested a reduction in the rate of AAA formation and growth, no benefit was observed with on the risk of AAA rupture. A recent study from Denmark reviewed a national ruptured AAA registry over 15 years starting in the late $1990 \mathrm{~s}(\mathrm{n}=362)$. In their series, $22.4 \%$ of ruptures were long-term metformin users (compared to $28.8 \%$ of controls). After adjustment for covariates, the OR reported was 0.84 (CI, $0.61-1.17)$ suggesting no effect of metformin in the diabetic population at rupture risk reduction.(35) The authors conclude their findings, in conjunction with previous studies establishing the effectiveness of all antidiabetic medications equally(36), suggest that DM, rather than metformin, is the overwhelming variable generating protection to AAA. The data presented in this manuscript can be interpreted to provide limited evidence supporting that assertion. However, it is worth noting that with exception to Treg (higher in nondiabetics) and Th17 expression (lower in diabetics), no differences were noted in the markers reported in this manuscript when the RFM cohort was compared to diabetics regardless of metformin status as well.

There are several limitations to the results reported in this study. Because of the design of this investigation, a retrospective review of a prospectively maintained database, small numbers of patients 
were included in several of the groups for statistical analysis. This limits the power, amplifies error, and makes conclusions weak. Additionally, the vast majority of samples in our biorepository was collected from patients at our VA medical facility; therefore, nearly $100 \%$ of the subjects studied were male further limiting the generalizability of the data presented. 


\section{Conclusion}

Although metformin has been associated with protection against AAA in previous studies, we did not observe any alterations in cytokine signaling, $\mathrm{CD}^{+}$phenotype expression, or circulating antigens/antibodies which would explain this effect. 


\section{References}

1. Lederle FA, Johnson GR, Wilson SE, Chute EP, Littooy FN, Bandyk D, et al. Prevalence and associations of abdominal aortic aneurysm detected through screening. Aneurysm Detection and Management (ADAM) Veterans Affairs Cooperative Study Group. Annals of internal medicine. 1997;126(6):441-9.

2. Ailawadi G, Eliason JL, Upchurch GR, Jr. Current concepts in the pathogenesis of abdominal aortic aneurysm. Journal of vascular surgery. 2003;38(3):584-8.

3. Collins MJ, Bersi M, Wilson E, Humphrey JD. Mechanical properties of suprarenal and infrarenal abdominal aorta: implications for mouse models of aneurysms. Medical engineering \& physics. 2011;33(10):1262-9.

4. Green LA, Wang SK, Kusumanchi P, Zhang L, C.C. B, Murphy MP. Defects in Tr1 Cell Immune Regulation are Associated with Abdominal Aortic Aneurysm Pathogenesis (In Review). Circulation research. 2017.

5. Glimaker H, Holmberg L, Elvin A, Nybacka O, Almgren B, Bjorck CG, et al. Natural history of patients with abdominal aortic aneurysm. European journal of vascular surgery. 1991;5(2):125-30.

6. Lindholt JS, Sogaard R, Laustsen J. Prognosis of ruptured abdominal aortic aneurysms in Denmark from 1994-2008. Clinical epidemiology. 2012;4:111-3.

7. Ashton HA, Buxton MJ, Day NE, Kim LG, Marteau TM, Scott RA, et al. The Multicentre Aneurysm Screening Study (MASS) into the effect of abdominal aortic aneurysm screening on mortality in men: a randomised controlled trial. Lancet. 2002;360(9345):1531-9.

8. Participants USAT. Mortality results for randomised controlled trial of early elective surgery or ultrasonographic surveillance for small abdominal aortic aneurysms. Lancet. 1998;352(9141):1649-55.

9. Aljabri B, Al Wahaibi K, Abner D, Mackenzie KS, Corriveau MM, Obrand DI, et al. Patientreported quality of life after abdominal aortic aneurysm surgery: a prospective comparison of endovascular and open repair. Journal of vascular surgery. 2006;44(6):1182-7.

10. Lederle FA, Johnson GR, Wilson SE, Littooy FN, Krupski WC, Bandyk D, et al. Yield of repeated screening for abdominal aortic aneurysm after a 4-year interval. Aneurysm Detection and Management Veterans Affairs Cooperative Study Investigators. Archives of internal medicine. 2000;160(8):1117-21.

11. Lederle FA. The strange relationship between diabetes and abdominal aortic aneurysm. European journal of vascular and endovascular surgery : the official journal of the European Society for Vascular Surgery. 2012;43(3):254-6.

12. De Rango P, Farchioni L, Fiorucci B, Lenti M. Diabetes and abdominal aortic aneurysms. European journal of vascular and endovascular surgery : the official journal of the European Society for Vascular Surgery. 2014;47(3):243-61.

13. Dinesh Shah A, Langenberg C, Rapsomaniki E, Denaxas S, Pujades-Rodriguez M, Gale CP, et al. Type 2 diabetes and incidence of a wide range of cardiovascular diseases: a cohort study in 1.9 million people. Lancet. 2015;385 Suppl 1:S86.

14. Fujimura N, Xiong J, Kettler EB, Xuan H, Glover KJ, Mell MW, et al. Metformin treatment status and abdominal aortic aneurysm disease progression. Journal of vascular surgery. 2016;64(1):4654.e8.

15. Hinchliffe RJ. Metformin and Abdominal Aortic Aneurysm. European journal of vascular and endovascular surgery : the official journal of the European Society for Vascular Surgery. 2017.

16. Wang SK, Green LA, Gutwein AR, Drucker NA, Motaganahalli RL, Fajardo A, et al. Rationale and Design of the ARREST Trial Investigating Mesenchymal Stem Cells in the Treatment of Small AAA (In Press). Annals of vascular surgery. 2017.

17. Wang SK, Green LA, Gutwein AR, Gupta AK, Babbey CC, Motaganahalli RL, et al. Osteopontin May be a Driver of Abdominal Aortic Aneurysm Formation. Journal of vascular surgery. 2017. 
18. Wang SK, Green LA, Gutwein AR, Motaganahalli RL, Fajardo A, Sawchuk AP, et al. AAA is Characterized by Abberations in Both Immune Regulation and Inflammation (In Press). Surgery. 2018. 19. Wang SK, Xie J, Green LA, McCready RA, Motaganahalli RL, Fajardo A, et al. TSG-6 is Highly Expressed in Human Abdominal Aortic Aneurysms (In Press). J Surg Res. 2017.

20. LeFevre ML. Screening for abdominal aortic aneurysm: U.S. Preventive Services Task Force recommendation statement. Annals of internal medicine. 2014;161(4):281-90.

21. Lee SH, Goswami S, Grudo A, Song LZ, Bandi V, Goodnight-White S, et al. Antielastin autoimmunity in tobacco smoking-induced emphysema. Nature medicine. 2007;13(5):567-9.

22. Takagi H, Umemoto T. A contemporary meta-analysis of the association of diabetes with abdominal aortic aneurysm. International angiology : a journal of the International Union of Angiology. 2015;34(4):375-82.

23. Miyama N, Dua MM, Yeung JJ, Schultz GM, Asagami T, Sho E, et al. Hyperglycemia limits experimental aortic aneurysm progression. Journal of vascular surgery. 2010;52(4):975-83.

24. Climent E, Benaiges D, Chillaron JJ, Flores-Le Roux JA, Pedro-Botet J. Diabetes mellitus as a protective factor of abdominal aortic aneurysm: Possible mechanisms. Clinica e investigacion en arteriosclerosis : publicacion oficial de la Sociedad Espanola de Arteriosclerosis. 2018.

25. Hsu CY, Su YW, Chen YT, Tsai SH, Chang CC, Li SY, et al. Association between use of oralantidiabetic drugs and the risk of aortic aneurysm: a nested case-control analysis. Cardiovascular diabetology. 2016;15(1):125.

26. Golledge J, Moxon J, Pinchbeck J, Anderson G, Rowbotham S, Jenkins J, et al. Association between metformin prescription and growth rates of abdominal aortic aneurysms. The British journal of surgery. 2017;104(11):1486-93.

27. Pernicova I, Korbonits M. Metformin--mode of action and clinical implications for diabetes and cancer. Nature reviews Endocrinology. 2014;10(3):143-56.

28. An H, Wei R, Ke J, Yang J, Liu Y, Wang X, et al. Metformin attenuates fluctuating glucoseinduced endothelial dysfunction through enhancing GTPCH1-mediated eNOS recoupling and inhibiting NADPH oxidase. Journal of diabetes and its complications. 2016;30(6):1017-24.

29. Esfahanian N, Shakiba Y, Nikbin B, Soraya H, Maleki-Dizaji N, Ghazi-Khansari M, et al. Effect of metformin on the proliferation, migration, and MMP-2 and -9 expression of human umbilical vein endothelial cells. Molecular medicine reports. 2012;5(4):1068-74.

30. Isoda K, Young JL, Zirlik A, MacFarlane LA, Tsuboi N, Gerdes N, et al. Metformin inhibits proinflammatory responses and nuclear factor-kappaB in human vascular wall cells. Arteriosclerosis, thrombosis, and vascular biology. 2006;26(3):611-7.

31. Vasamsetti SB, Karnewar S, Kanugula AK, Thatipalli AR, Kumar JM, Kotamraju S. Metformin inhibits monocyte-to-macrophage differentiation via AMPK-mediated inhibition of STAT3 activation: potential role in atherosclerosis. Diabetes. 2015;64(6):2028-41.

32. Groux H, O'Garra A, Bigler M, Rouleau M, Antonenko S, de Vries JE, et al. A CD4+ T-cell subset inhibits antigen-specific T-cell responses and prevents colitis. Nature. 1997;389(6652):737-42. 33. Dale MA, Xiong W, Carson JS, Suh MK, Karpisek AD, Meisinger TM, et al. Elastin-Derived Peptides Promote Abdominal Aortic Aneurysm Formation by Modulating M1/M2 Macrophage Polarization. Journal of immunology (Baltimore, Md : 1950). 2016;196(11):4536-43.

34. Daugherty A, Cassis LA. Mechanisms of abdominal aortic aneurysm formation. Current atherosclerosis reports. 2002;4(3):222-7.

35. Kristensen KL, Pottegard A, Hallas J, Rasmussen LM, Lindholt JS. Metformin treatment does not affect the risk of ruptured abdominal aortic aneurysms. Journal of vascular surgery. 2017;66(3):76874.e2.

36. Thompson A, Cooper JA, Fabricius M, Humphries SE, Ashton HA, Hafez H. An analysis of drug modulation of abdominal aortic aneurysm growth through 25 years of surveillance. Journal of vascular surgery. 2010;52(1):55-61.e2. 